\title{
An Application of Similarity Measure of Intuitionistic Fuzzy Soft Sets Based on Set Theoretical Approach
}

\author{
Dr. N. Sarala ${ }^{1}$, B. Suganya ${ }^{2}$ \\ ${ }^{1}$ Department of Mathematics, A.D.M College for women, Nagapattinam (India)
}

${ }^{2}$ Department of Mathematics, Bharathidasan University Constituent College of arts and science, Nagapattinam (India)

\begin{abstract}
In this article, the concept of intuitionistic fuzzy soft sets and some of their properties are discussed. Some results are proved. Then the concept of similarity measure for intuitionistic fuzzy soft sets based on set theoretic approach between two intuitionistic fuzzy soft sets, some examples and basic properties is also studied. An algorithm is developed in intuitionistic fuzzy soft set and an example is given to illustrate possible application in a decision making problem.
\end{abstract}

Keywords: Soft Set, Fuzzy Soft Set, Intuitionistic Fuzzy Set, Intuitionistic Fuzzy Soft Set, Similarity measures

\section{Introduction}

To solve complex problems in economy, engineering, environmental science and social science, the methods in classical mathematics may not be successfully modeled because of various types of uncertainties. There are some mathematical theories for dealing with uncertainties such as; fuzzy set theory, soft set theory, fuzzy soft set theory and so on.

In many complicated problems arising in the fields of engineering, social science, economics, medical science etc involving uncertainties, classical methods are found to be inadequate in recent times. Molodtsov initiated soft set theory [12] as a new mathematical tool for dealing with uncertainties. In recent times, researchers have contributed a lot towards fuzzification of soft set theory.

Zadeh[13] introduced fuzzy set theory to represent uncertainty and vagueness mathematically with formalized logical tools for dealing with the imprecision inherent in many real world problems. . In fuzzy set theory, the membership of an element to a fuzzy set is a single value between zero and one. However in reality, it may not always be true that the degree of non-membership of an element in a fuzzy set is equal to 1 minus the membership degree because there may be some hesitation degree Again Atanassov[1] generalised the fuzzy set by defining the intuitionistic fuzzy to many situations where fuzzy set theory cannot be suitably applied but intuitionistic fuzzy set theory can be used for a fair analysis. Maji et al.[7,8,9] have further studied the theory of soft sets introduced by Molodtsov[12] and introduced the concept of fuzzy soft sets and intuitionistic fuzzy soft sets[4].

In this paper, we first present the basic definitions of soft sets, fuzzy sets ,fuzzy soft sets , Intuitionistic Fuzzy Set, Intuitionistic Fuzzy Soft Set that are useful for subsequent discussions and study their related properties.
Throughout this work, $U$ refers to the initial universe, $P(U)$ is the power set of $U, E$ is a set of parameters and $A \subseteq E . S(U)$ denotes the set of all soft sets over $U$.

\section{Preliminaries}

In this section, we have presented the basic definition related to fuzzy sets, soft sets, and fuzzy soft sets which would be used in the sequel.

\section{Definition 2.1. Soft set}

Suppose that $U$ is an initial universe set and $E$ is a set of parameters, let $\mathrm{P}(\mathrm{U})$ denotes the power set of $\mathrm{U}$.A pair $(\mathrm{F}, \mathrm{E})$ is called a soft set over $U$ where $F$ is a mapping given by $\mathrm{F}: \mathrm{E} \rightarrow \mathrm{P}(\mathrm{U})$.

Clearly, a soft set is a mapping from parameters to $\mathrm{P}(\mathrm{U})$, and it is not a set, but a parameterized family of subsets of the Universe.

\section{Definition 2.2. Fuzzy soft set}

Let $U$ be an initial Universe set and $E$ be the set of parameters. Let $\mathrm{A} \subset \mathrm{E}$. A pair (F, A) is called fuzzy soft set (FSS) over $U$ where $F$ is a mapping given by $F$ : $A \rightarrow I^{U}$, where $\mathrm{I}^{\mathrm{U}}$ denotes the collection of all fuzzy subsets of $\mathrm{U}$.

\section{Definition 2.3. Fuzzy soft class}

Let $\mathrm{U}$ be an initial Universe set and $\mathrm{E}$ be the set of attributes. Then the pair $(\mathrm{U}, \mathrm{E})$ denotes the collection of all fuzzy soft sets on $U$ with attributes from $E$ and is called a fuzzy soft class.

\section{Some Results of Intuitionistic Fuzzy Soft Sets}

In this section, we presented the basic concepts of intuitionistic fuzzy soft set theory which are useful for subsequent discussions, and then we discuss basic algebraic structures of intuitionistic fuzzy soft set and discuss some related properties. 


\section{International Journal of Science and Research (IJSR) \\ ISSN (Online): 2319-7064 \\ Index Copernicus Value (2013): 6.14 | Impact Factor (2015): 6.391}

Let $U$ be an initial universe, and $E$ be the set of all possible parameters under consideration with respect to $U$. The set of all subsets of $U$, i.e. the power set of $U$ is denoted by $P(U)$ and the set of all Intuitionistic fuzzy subsets of $U$ is denoted by $I F^{U}$. Let $A$ be a subset of $E$.

\section{Definition 3.1[10]}

An intuitionistic fuzzy set $A$ over the universe $U$ can be defined as follows

$$
A=\left\{\left(x, \mu_{A}(x), v_{A}(x): x \in U\right\},\right.
$$

where $\mu_{A}(x): U \rightarrow[0,1], v_{A}(x): U \rightarrow[0,1]$ with the property $0 \leq$ $\mu_{A}(x)+v_{A}(x) \leq 1 \quad \forall x \in U$. The values $\mu_{A}(x)$ and $v_{A}(x)$ represent the degree of membership and non-membership of $x$ to $A$ respectively. $\pi_{A}(x)=1-\left(\mu_{A}(x)+v_{A}(x)\right)$ is called the intuitionistic fuzzy index.

\section{Definition $3.2[10]$}

An intuitionistic fuzzy set $A$ over the universe $U$ defined as $A$ $=\{(x, 0,1): x \in U\}$ is said to be intuitionistic fuzzy null set and is denoted by 0 .

\section{Definition 3.3 [10]}

An intuitionistic fuzzy set $A$ over the universe $U$ defined as $A$ $=\{(x, 1,0): x \in U\}$ is said to be intuitionistic fuzzy absolute set and is denoted by 1 .

\section{Definition 3.4 [11]}

Let $A=\left\{\left(x, \mu_{A}(x), v_{A}(x): x \in U\right\}\right.$ be an intuitionistic fuzzy set over the universe $U$, where $\mu_{A}(x): U \rightarrow[0,1], v_{A}(x): U \rightarrow[0,1]$ with the property $0 \leq \mu_{A}(x)+v_{A}(x) \leq 1 \forall x \in U$ Complement of $\boldsymbol{A}$ is denoted by $A^{c}$ and defined as the intuitionistic fuzzy set

$$
A^{c}=\left\{\left(x, v_{A}(x), \mu_{A}(x): x \in U\right\} .\right.
$$

\section{Definition 3.5 [11]}

Let $A=\left\{\left(x, \mu_{A}(x), v_{A}(x): x \in U\right\}, B=\left\{\left(x, \mu_{B}(x), v_{B}(x): x \in U\right\}\right.\right.$ be two intuitionistic fuzzy sets over the universe $U$, where $\mu_{A}(x): U \rightarrow[0,1], v_{A}(x): U \rightarrow[0,1], \mu_{B}(x): U \rightarrow[0,1], v_{B}(x): U$ $\rightarrow[0,1]$ with the property $0 \leq \mu_{A}(x)+v_{A}(x), \mu_{B}(x)+v_{B}(x) \leq$ $1, \forall x \in U$.

Union of $A$ and $B$ is defined as $A \cup B=\left\{x, \max \left(\mu_{A}(x), \mu_{B}(x)\right), \min \left(v_{A}(x), v_{B}(x)\right)\right\}$ and

Intersection of $A$ and $B$ is defined as $A \cap B=\left\{x, \min \left(\mu_{A}(x), \mu_{B}(x)\right), \max \left(v_{A}(x), v_{B}(x)\right)\right\}$

\section{Definition 3.6 [10]}

Let $U$ be an initial universe set and $E$ be the set of parameters. Let $I F^{U}$ denote the collection of all intuitionistic fuzzy subsets of $U$. Let $A \subseteq E$. A pair $(F, A)$ is called an intuitionistic fuzzy soft set over $U$ where $F$ is a mapping given by $F: A \rightarrow I F^{U}$.

\section{Definition 3.7 [10]}

Union of two intuitionistic fuzzy soft sets $(F, A)$ and $(G, B)$ over $(U, E)$ is an Intuitionistic fuzzy soft set $(H, C)$, where $C=A \cup B$ and $\forall \varepsilon \in \mathrm{C}$,

$$
\mathrm{H}(\varepsilon)= \begin{cases}\mathrm{F}(\varepsilon), & \text { if } \varepsilon \in A-B \\ \mathrm{G}(\varepsilon), & \text { if } \varepsilon \in \mathrm{B}-\mathrm{A} \\ \mathrm{F}(\varepsilon) \cup \mathrm{G}(\varepsilon), & \text { if } \varepsilon \in A \cap \mathrm{B}\end{cases}
$$

and is written as $(\mathrm{F}, \mathrm{A}) \widetilde{\cup}(\mathrm{G}, \mathrm{B})=(\mathrm{H}, \mathrm{C})$

\section{Definition 3.8 [11]}

Let $(F, A)$ and $(G, B)$ be two intuitionistic fuzzy soft sets over $(U, E)$. Then intersection $(F, A)$ and $(G, B)$ is an intuitionistic fuzzy soft set $(H, C)$, where $C=A \cap B$ and $\forall \varepsilon \in \mathrm{C}$, $\mathrm{H}(\varepsilon)=\mathrm{F}(\varepsilon) \tilde{\cap} \mathrm{G}(\varepsilon)$.

We write $(\mathrm{F}, \mathrm{A}) \tilde{\cap}(\mathrm{G}, \mathrm{B})=(\mathrm{H}, \mathrm{C})$.

Definition 3.9 [5]

For two Intuitionistic fuzzy soft sets $(F, A)$ and $(G, B)$ over $(U, E)$, we say that $(F, A)$ is an intuitionistic fuzzy soft subset of $(G, B)$, if

(i) $A \subseteq B$,

(ii) For all $\varepsilon \in A, \mathrm{~F}(\varepsilon) \subseteq \mathrm{G}(\varepsilon)$.

and is written as $(F, A) \subseteq(G, B)$.

\section{Definition 3.10}

The complement of an Intuitionistic fuzzy soft set $(F, A)$ is denoted by $(F, A)^{\mathrm{c}}$ and is defined by $(F, A)^{\mathrm{c}}=\left(F^{\mathrm{c}}, A\right)$, where $F^{c:} A \rightarrow I F^{U}$ is a mapping given by $F^{\mathrm{c}}(\varepsilon)=(F(\varepsilon))^{\mathrm{c}}$ for all $\varepsilon \in A$. Thus if $F(\varepsilon)=\left\{\left(x, \mu_{F(\varepsilon)}(x), v_{F(\varepsilon)}(x): x \in U\right\}\right.$, then for all $\varepsilon \in A$, $F^{\mathrm{c}}(\varepsilon)=(F(\varepsilon))^{\mathrm{c}}=\left\{\left(x, \mu_{F(\varepsilon)}(x), v_{F(\varepsilon)}(x): x \in U\right\}\right.$

Basic results:

[Addition]: $\begin{aligned}(\mathrm{F}: \mathrm{A}) \oplus(\mathrm{G}: \mathrm{B})=\{( & \left(x, \mu_{F(\varepsilon)}(x)+\mu_{G(\varepsilon)}(x)-\mu_{F(\varepsilon)}(x)\right. \\ & \left.\left.\left.\mu_{G(\varepsilon)}(x), v_{F(\varepsilon)}(x), v_{G(\varepsilon)}(x)\right)\right)\right\}\end{aligned}$

[Multiplication]:

$(\mathrm{F}: \mathrm{A}) \otimes(\mathrm{G}: \mathrm{B})=\left\{\left(x, \mu_{F(\varepsilon)}(x) \mu_{G(\varepsilon)}(x), v_{F(\varepsilon)}(x)\right.\right.$

$$
\left.\left.\left.+v_{G(\varepsilon)}(x)-v_{F(\varepsilon)}(x) v_{G(\varepsilon)}(x)\right)\right)\right\}
$$

[Difference]:

$(\mathrm{F}: \mathrm{A})-(\mathrm{G}: \mathrm{B})=\left\{\left(x, \min \left(\mu_{F(\varepsilon)}(x), v_{G(\varepsilon)}(x)\right)\right.\right.$, $\left.\left.\max \left(v_{F(\varepsilon)}(x), \mu_{G(\varepsilon)}(x)\right)\right)\right\}, \forall x \in U$

[Equality]:

$(\mathrm{F}, \mathrm{A})=(\mathrm{G}, \mathrm{B}) \leftrightarrow \mu_{F(\varepsilon)}(x)=\mu_{G(\varepsilon)}(x)$ and $v_{F(\varepsilon)}(x)=v_{G(\varepsilon)}(x), \forall x \in U$

[Complement]:

$(\mathrm{F}, \mathrm{A})^{\mathrm{c}}=\left\{\left(x, v_{F(\varepsilon)}(x), \mu_{F(\varepsilon)}(x)\right), x \in U\right\}$

[union]:

$(\mathrm{F}, \mathrm{A}) \cup(\mathrm{G}, \mathrm{B})=\left\{\left(x, \max \left(\mu_{F(\varepsilon)}(x), \mu_{G(\varepsilon)}(x)\right)\right.\right.$, $\left.\min \left(v_{F(\varepsilon)}(x), v_{G(\varepsilon)}(x)\right)\right\}$

[intersection]:

$(\mathrm{F}: \mathrm{A}) \cap(\mathrm{G}: \mathrm{B})=\left\{\left(x, \min \left(\mu_{F(\varepsilon)}(x), \mu_{G(\varepsilon)}(x)\right)\right.\right.$, $\left.\max \left(v_{F(\varepsilon)}(x), v_{G(\varepsilon)}(x)\right)\right\}$ 


\section{International Journal of Science and Research (IJSR) \\ ISSN (Online): 2319-7064}

Index Copernicus Value (2013): 6.14 | Impact Factor (2015): 6.391

[Cartesian product]:

$(\mathrm{F}: \mathrm{A}) \times(\mathrm{G}: \mathrm{B})=\left\{\left(x, \mu_{F(\varepsilon)}(x) \mu_{G(\varepsilon)}(x)\right)\right.$,

$$
\left.\left.v_{F(\varepsilon)}(x) v_{G(\varepsilon)}(x)\right)\right\}, \forall x \in U
$$

[ inclusion]:

$$
\begin{aligned}
&(\mathrm{F}: \mathrm{A}) \subseteq(\mathrm{G}: \mathrm{B}) \leftrightarrow \mu_{F(\varepsilon)}(x) \leq \mu_{G(\varepsilon)}(x) \text { and } \\
& v_{F(\varepsilon)}(x) \geq v_{G(\varepsilon)}(x), \forall x \in U
\end{aligned}
$$

\section{Theorem: 3.11( union)}

Let (F:A),and (G:B) be any two intuitionistic fuzzy soft sets over (U:E), then Prove That $(\mathrm{F}: \mathrm{A}) \tilde{\cup}(\mathrm{G}: \mathrm{B})=(\mathrm{G}: \mathrm{B}) \tilde{\cup}(\mathrm{F}: \mathrm{A})$

\section{Proof:}

(i) Let (F:A) $\widetilde{\cup}(\mathrm{G}: \mathrm{B})=(\mathrm{H}, \mathrm{C})$ where $\mathrm{C}=\mathrm{A} \cup \mathrm{B}$ and $\forall \varepsilon \in$ C,

$$
\mathrm{H}(\varepsilon)=\left\{\begin{array}{l}
\left\{\left(x, \mu_{E(z)}(x), v_{F(z)}(x): x \in U\right\}, \text { if } \varepsilon \in \mathrm{A}-\mathrm{B}\right. \\
\left\{\left(x, \mu_{G(z)}(x), v_{G(z)}(x) ; x \in U\right\}, \text { if } \varepsilon \in \mathrm{B}-\mathrm{A}\right. \\
\max \left(\mu_{\mathbb{R}(z)}(x), \mu_{G(z)}(x)\right), \\
\left.\min \left(v_{\left.E_{(z)}\right)}(x), v_{G(z)}(x)\right)\right\}, \text { if } \varepsilon \in \mathrm{A} \cap \mathrm{B}
\end{array}\right.
$$

Again Let $(\mathrm{G}: \mathrm{B}) \widetilde{\cup}(\mathrm{F}: \mathrm{A})=(\mathrm{H}, \mathrm{C})$ where $\mathrm{C}=\mathrm{A} \cup \mathrm{B}$ and $\forall$ $\varepsilon \in \mathrm{C}$,

$$
\mathrm{H}(\varepsilon)=\left\{\begin{array}{c}
\left\{\left(x, \mu_{G(s)}(x), v_{G(s)}(x): x \in U\right\},\right. \\
\text { if } \varepsilon \in \mathrm{B}-\mathrm{A} \\
\left\{\left(x, \mu_{F(s)}(x), v_{F(s)}(x): x \in U\right\},\right. \\
\text { if } \varepsilon \in \mathrm{A}-\mathrm{B} \\
\left\{x, \max \left(\mu_{F(s)}(x), \mu_{G(s)}(x)\right),\right. \\
\left.\min \left(v_{F(s)}(x), v_{G(s)}(x)\right)\right\}, \\
\text { if } \varepsilon \in \mathrm{A} \cap \mathrm{B}
\end{array}\right.
$$

Hence $(\mathrm{F}: \mathrm{A}) \tilde{\cup}(\mathrm{G}: \mathrm{B})=(\mathrm{G}: \mathrm{B}) \tilde{\cup}(\mathrm{F}: \mathrm{A})$

\section{Theorem: 3.12(intersection)}

Let (F:A), and (G:B) be any two intuitionistic fuzzy soft sets over (U:E), then Prove That $(\mathrm{F}: \mathrm{A}) \tilde{\cap}(\mathrm{G}: \mathrm{B})=(\mathrm{G}: \mathrm{B}) \tilde{\cap}$ (F:A)

\section{Proof:}

Let $(\mathrm{F}: \mathrm{A}) \tilde{\cap}(\mathrm{G}: \mathrm{B})=(\mathrm{H}, \mathrm{C})$ where $\mathrm{C}=\mathrm{A} \cap \mathrm{B}$ and $\forall \varepsilon \in \mathrm{C}$,

$$
\begin{aligned}
\mathrm{H}(\varepsilon)= & \mathrm{F}(\varepsilon) \cap \mathrm{G}(\varepsilon) \\
= & \left\{x, \min \left(\mu_{F(\varepsilon)}(x), \mu_{G(\varepsilon)}(x)\right),\right. \\
& \left.\max \left(v_{F(\varepsilon)}(x), v_{G(\varepsilon)}(x)\right)\right\}
\end{aligned}
$$

Again Let (G: B) $\tilde{\cap}(\mathrm{F}: \mathrm{A})=(\mathrm{H}, \mathrm{C})$

where $\mathrm{C}=\mathrm{A} \cap \mathrm{B}$ and $\forall \varepsilon \in \mathrm{C}$,

$$
\begin{aligned}
\mathrm{H}(\varepsilon)= & \mathrm{G}(\varepsilon) \cap \mathrm{F}(\varepsilon) \\
= & \left\{x, \min \left(\mu_{F(\varepsilon)}(x), \mu_{G(\varepsilon)}(x)\right),\right. \\
& \left.\quad \max \left(v_{F(\varepsilon)}(x), v_{G(\varepsilon)}(x)\right)\right\}
\end{aligned}
$$

Hence $(F: A) \tilde{\cap}(\mathrm{G}: B)=(\mathrm{G}: \mathrm{B}) \tilde{\cap}(\mathrm{F}: \mathrm{A})$

\section{Theorem: 3.13(difference)}

Let (F:A), and (G:B) be any two intuitionistic fuzzy soft sets over (U:E), then Prove That

(i) $(\mathrm{F}: \mathrm{A})-(\mathrm{G}: \mathrm{B})=(\mathrm{F}: \mathrm{A}) \cap(\mathrm{G}: \mathrm{B})^{\mathrm{c}}$

(ii) $(\mathrm{F}: \mathrm{A})-(\mathrm{G}: \mathrm{B})=(\mathrm{G}: \mathrm{B})-(\mathrm{F}: \mathrm{A})$ iff $(\mathrm{F}: \mathrm{A})=(\mathrm{G}: \mathrm{B})$

(iii) $(\mathrm{F}: \mathrm{A})-(\mathrm{G}: \mathrm{B})=(\mathrm{G}: \mathrm{B})^{\mathrm{c}}-(\mathrm{F}: \mathrm{A})^{\mathrm{c}}$
Proof:

(i) Let $F(\varepsilon)=\left\{\left(x, \mu_{F(\varepsilon)}(x), v_{F(\varepsilon)}(x): x \in U\right\}\right.$

and $G(\varepsilon)=\left\{\left(x, \mu_{G(\varepsilon)}(x), \nu_{G(\varepsilon)}(x): x \in U\right\}\right.$,

then

$F(\varepsilon)-G(\varepsilon)=\left\{\left(x, \min \left(\mu_{F(\varepsilon)}(x), v_{G(\varepsilon)}(x)\right)\right.\right.$,

But $\left.\left.\max \left(v_{F(\varepsilon)}(x), \mu_{G(\varepsilon)}(x)\right)\right)\right\}, \forall x \in U$

$$
(G(\varepsilon))^{\mathrm{c}}=\left\{\left(x, v_{G(\varepsilon)}(x), \mu_{G(\varepsilon)}(x): x \in U\right\}\right.
$$

$\Rightarrow$

$\mathrm{F}(\varepsilon) \cap(\mathrm{G}(\varepsilon))^{\mathrm{c}}=\left\{\left(x, \min \left(\mu_{F(\varepsilon)}(x), v_{G(\varepsilon)}(x)\right)\right.\right.$,

Since $\left.\left.\max \left(v_{F(\varepsilon)}(x), \mu_{G(\varepsilon)}(x)\right)\right)\right\}, \forall x \in U$

$\mathrm{F}(\varepsilon) \cap \mathrm{G}(\varepsilon)=\left\{x, \min \left(\mu_{F(\varepsilon)}(x), \mu_{G(\varepsilon)}(x)\right)\right.$, $\left.\max \left(v_{F(\varepsilon)}(x), v_{G(\varepsilon)}(x)\right)\right\}$

(ii) $F(\varepsilon)-G(\varepsilon)=\left\{\left(x, \min \left(\mu_{F(\varepsilon)}(x), v_{G(\varepsilon)}(x)\right)\right.\right.$, $\left.\left.\max \left(v_{F(\varepsilon)}(x), \mu_{G(\varepsilon)}(x)\right)\right)\right\}, \forall x \in U$

$$
\text { if } \begin{aligned}
F(\varepsilon)=G(\varepsilon) \Rightarrow \mu_{F(\varepsilon)}(x)=\mu_{G(\varepsilon)}(x) \\
\text { and } \\
v_{F(\varepsilon)}(x)=v_{G(\varepsilon)}(x)
\end{aligned}
$$

From this it is certain that

$$
G(\varepsilon)-F(\varepsilon)=F(\varepsilon)-G(\varepsilon)
$$

and the result follows.

(iii) $F(\varepsilon)-G(\varepsilon)=\left\{\left(x, \min \left(\mu_{F(\varepsilon)}(x), v_{G(\varepsilon)}(x)\right)\right.\right.$, $\left.\left.\max \left(v_{F(\varepsilon)}(x), \mu_{G(\varepsilon)}(x)\right)\right)\right\}, \forall x \in U$

w.k.t

$(F(\varepsilon))^{\mathrm{c}}=\left\{\left(x, v_{F(\varepsilon)}(x), \mu_{F(\varepsilon)}(x): x \in U\right\}\right.$ and

$(G(\varepsilon))^{\mathrm{c}}=\left\{\left(x, v_{G(\varepsilon)}(x), \mu_{G(\varepsilon)}(x): x \in U\right\}\right.$

$\Rightarrow$

$(G(\varepsilon))^{\mathrm{c}}-(F(\varepsilon))^{\mathrm{c}}=\left\{\left(x, \min \left(v_{G(\varepsilon)}(x), \mu_{F(\varepsilon)}(x)\right)\right.\right.$, $\left.\left.\max \left(\mu_{G(\varepsilon)}(x), v_{F(\varepsilon)}(x),\right)\right)\right\}, \forall x \in \mathrm{U}$ and the result is straight forward.

\section{Theorem :3.14(idempotent property)}

Let (F:A) be intuitionistic fuzzy soft sets over (U:E), then Prove That

(i) $(\mathrm{F}: \mathrm{A}) \tilde{\cap}(\mathrm{F}: \mathrm{A})=(\mathrm{F}: \mathrm{A})$

(ii) $(\mathrm{F}: \mathrm{A}) \widetilde{\cup}(\mathrm{F}: \mathrm{A})=(\mathrm{F}: \mathrm{A})$.

\section{Proof}

(i) Let $(\mathrm{F}: \mathrm{A}) \tilde{\cap}(\mathrm{F}: \mathrm{A})=(\mathrm{F}: \mathrm{A})$ where $\mathrm{C}=\mathrm{A} \cap \mathrm{A}$ and $\forall \varepsilon \in \mathrm{C}$,

$\mathrm{H}(\varepsilon)=\mathrm{F}(\varepsilon) \cap \mathrm{F}(\varepsilon)=\mathrm{F}(\varepsilon)$

$=\left\{x, \min \left(\mu_{F(\varepsilon)}(x), \mu_{F(\varepsilon)}(x)\right)\right.$, $\left.\max \left(v_{F(\varepsilon)}(x), v_{F(\varepsilon)}(x)\right)\right\}$

$\left.=\left\{x, \mu_{F(\varepsilon)}(x), v_{F(\varepsilon)}(x)\right\}: x \in U\right\}$

$=\mathrm{F}(\varepsilon)$

Thus $(\mathrm{F}: \mathrm{A}) \tilde{\cap}(\mathrm{F}: \mathrm{A})=(\mathrm{F}: \mathrm{A})$

(ii)Let $(\mathrm{F}: \mathrm{A}) \sim(\mathrm{F}: \mathrm{A})=(\mathrm{F}: \mathrm{A})$ where $\mathrm{C}=\mathrm{A} \cup \mathrm{A}$ and $\forall \varepsilon \in \mathrm{C}$,

$$
\mathrm{H}(\varepsilon)=\mathrm{F}(\varepsilon) \cup \mathrm{F}(\varepsilon)=\mathrm{F}(\varepsilon)
$$

Thus $(\mathrm{F}: \mathrm{A}) \widetilde{\cup}(\mathrm{F}: \mathrm{A})=(\mathrm{F}: \mathrm{A})$

\section{Theorem :3.15(absorption property)}

Let (F:A) and (G:B) be any two intuitionistic fuzzy soft sets over (U:E), then Prove That

(i) $(\mathrm{F}: \mathrm{A}) \tilde{\cap}((\mathrm{F}: \mathrm{A}) \tilde{\cup}(\mathrm{G}: \mathrm{B}))=(\mathrm{F}: \mathrm{A})$

\section{Volume 5 Issue 6, June 2016}


International Journal of Science and Research (IJSR)

ISSN (Online): 2319-7064

Index Copernicus Value (2013): 6.14 | Impact Factor (2015): 6.391

(ii) $(\mathrm{F}: \mathrm{A}) \tilde{\cup}((\mathrm{F}: \mathrm{A}) \tilde{\cap}(\mathrm{G}: \mathrm{B}))=(\mathrm{F}: \mathrm{A})$

Proof: It is straight forward.

\section{Similarity Measure of Intuitionistic Fuzzy Soft Set [IFSS]:}

In this section, we give the definition of similarity measure for IFSS based on set theoretic approach with examples. Some properties are studied.

\section{Definitions: 4.1}

Let $\mathrm{U}=\left\{\mathrm{x}_{\mathrm{i}} ; \mathrm{i}=1,2, \ldots, \mathrm{m}\right\}$ be the universal set of elements and $\mathrm{E}=\left\{\mathrm{e}_{\mathrm{j}} ; \mathrm{j}=1,2, \ldots, \mathrm{n}\right\}$ be the set of parameters. Let $(\mathrm{F}, \mathrm{E})$ and $(\mathrm{G}, \mathrm{E})$ be two IFSS over $\mathrm{U}$. Then $(\mathrm{F}, \mathrm{E})=\left\{\mathrm{F}\left(\mathrm{e}_{\mathrm{j}}\right) \in \mathrm{IFSS}^{\mathrm{U}}, \mathrm{e}_{\mathrm{j}}\right.$ $\in E\}$ and $(G, E)=\left\{G\left(e_{j}\right) \in I_{F S S}{ }^{U}, e_{j} \in E\right\}$ where $F\left(e_{j}\right)$ is called the $e_{j}^{\text {th }}$ approzimation of $(F, E)$ and $G\left(e_{j}\right)$ is called the $e_{j}^{\text {th }}$ approzimation of $(\mathrm{G}, \mathrm{E})$ and $\mathrm{IFSS}^{\mathrm{U}}$ is the set of all IFSS on U. Let $M((F, E),(G, E))$ indicates the similarity measure between the IFSS (F,E) and (G,E). To find the similarity measure $(\mathrm{F}, \mathrm{E})$ and $(\mathrm{G}, \mathrm{E})$. We first have to find similarity between their $\mathrm{e}_{\mathrm{j}}^{\text {th }}$ approzimations.

Let $\mathrm{M}_{\mathrm{j}}((\mathrm{F}, \mathrm{E}),(\mathrm{G}, \mathrm{E}))$ denotes the similarity measure between the two $e_{j}^{\text {th }}$ approzimations of $F\left(e_{j}\right)$ and $G\left(e_{j}\right)$. The $M_{j}((F, E)$, $(\mathrm{G}, \mathrm{E}))$ is defined as follows

$$
\begin{array}{r}
\sum_{i=1}^{m} \sum_{j=1}^{n}\left|\mu_{F}\left(e_{i}\right)\left(x_{j}\right)-v_{F}\left(e_{i}\right)\left(x_{j}\right)\right| \wedge \\
M_{i}((F, E),(G, E))=\frac{\left|\mu_{G}\left(e_{j}\right)\left(x_{i}\right)-v_{G}\left(e_{j}\right)\left(x_{j}\right)\right|}{\sum_{i=1}^{m} \sum_{j=1}^{n}\left|\mu_{F}\left(e_{j}\right)\left(x_{j}\right)-v_{F}\left(e_{i}\right)\left(x_{j}\right)\right| v} \\
\left|\mu_{G}\left(e_{j}\right)\left(x_{i}\right)-v_{G}\left(e_{j}\right)\left(x_{j}\right)\right|
\end{array}
$$

\section{Theorem: 4.2}

If $\mathrm{M}((\mathrm{F}, \mathrm{E}),(\mathrm{G}, \mathrm{E}))$ be the similarity measure between two IFSS $(F, E)$ and $(\mathrm{G}, \mathrm{E})$ then

(i) $\mathrm{M}((\mathrm{F}, \mathrm{E}),(\mathrm{G}, \mathrm{E}))=\mathrm{M}((\mathrm{G}, \mathrm{E}),(\mathrm{F}, \mathrm{E}))$

(ii) $0 \leq \mathrm{M}((\mathrm{F}, \mathrm{E}),(\mathrm{G}, \mathrm{E})) \leq 1$

(iii) $\mathrm{M}((\mathrm{F}, \mathrm{E}),(\mathrm{G}, \mathrm{E}))=1$ iff $\quad(\mathrm{F}, \mathrm{E})=(\mathrm{G}, \mathrm{E})$

Proof: Obvious from the Definition

\section{Example: 4.3}

Let $\mathrm{U}=\left\{\mathrm{x}_{1}, \mathrm{x}_{2}, \mathrm{x}_{3}, \mathrm{x}_{4}\right\}$ be the universal set and $\mathrm{E}$ be the set parameters given by $E=\left\{e_{1}, e_{2}\right\}$. Let us consider two IFSS $(F$, E) and (GE) is given by

\begin{tabular}{|c|c|c|}
\hline$(\mathrm{F}, \mathrm{E})$ & $\mathrm{e}_{1}$ & $\mathrm{e}_{2}$ \\
\hline $\mathrm{x}_{1}$ & $(0.7,0.2)$ & $(0.6,0.4)$ \\
\hline $\mathrm{x}_{2}$ & $(0.9,0.0)$ & $(0.9,0.2)$ \\
\hline $\mathrm{x}_{3}$ & $(0.8,0.1)$ & $(0.6,0.3)$ \\
\hline $\mathrm{x}_{4}$ & $(0.6,0.4)$ & $(0.8,0.1)$ \\
\hline \multicolumn{3}{|c|}{ and } \\
\hline$(\mathrm{G}, \mathrm{E})$ & $\mathrm{e}_{1}$ & $\mathrm{e}_{2}$ \\
\hline $\mathrm{x}_{1}$ & $(0.6,0.2)$ & $(0.5,0.2)$ \\
\hline $\mathrm{x}_{2}$ & $(0.6,0.2)$ & $(0.7,0.1)$ \\
\hline $\mathrm{x}_{3}$ & $(0.5,0.4)$ & $(0.2,0.4)$ \\
\hline $\mathrm{x}_{4}$ & $(0.3,0.4)$ & $(0.8,0.2)$ \\
\hline
\end{tabular}

Now by equation (4.1) we have

$$
\begin{aligned}
& \sum_{i=1}^{m} \sum_{j=1}^{n}\left|\mu_{F}\left(e_{i}\right)\left(x_{j}\right)-v_{F}\left(e_{i}\right)\left(x_{j}\right)\right| \wedge \\
& \left|\mu_{G}\left(e_{i}\right)\left(x_{i}\right)-v_{G}\left(e_{i}\right)\left(x_{i}\right)\right| \\
& M_{i}((F, E),(G, E))=-v_{F}\left(e_{i}\right)\left(x_{j}\right) \mid v \\
& \sum_{j=1}^{m} \sum_{j=1}^{n}\left|\mu_{F}\left(e_{i}\right)\left(x_{j}\right)-\mu_{G}\left(e_{i}\right)\left(x_{i}\right)-v_{G}\left(e_{i}\right)\left(x_{i}\right)\right| \\
& M_{1}((F, E),(G, E))=0.43<1 / 2 \\
& M_{2}((F, E),(G, E))=0.8>1 / 2 \\
& M(F, G)=M a x\left\{M_{1}, M_{2}\right\}=M a x\{0.43,0.8\}=0.8 \\
& M(F, G)=\mathbf{0 . 8}
\end{aligned}
$$

\section{Application in Decision making problem}

In this section, we developed an algorithm based on similarity measure of two IFSS to Estimate the Best Apartment in town.

The Algorithm of this Method is as follows:

Step: 1

Construct an IFSS (F, E) over the Universe U.

Step:2

Construct IFSS (G, E) over the universe $U$ for the first Apartments.

Step:3

Construct IFSS (H, E) over the universe $U$ for the second Apartments.

\section{Step: 4}

Calculate similarity measure between $(\mathrm{F}, \mathrm{E})$ and $(\mathrm{G}, \mathrm{E})$; $(\mathrm{F}, \mathrm{E})$ and $(\mathrm{H}, \mathrm{E})$

\section{Step: 5}

If $M(F, G)>1 / 2$ then the person is possibly selected for Best apartment in town and if $M(F, G)<1 / 2$, then the person is not selected for Best apartment in town .

Here we are giving an example of a decision making method. The similarity measure of two IFSSs based on set theoretic approach can be applied to select whether a Best apartment in town or not.

Consider a apartment selection problem. Suppose there are 2 Apartments $\left\{a_{1}, a_{2}\right\}$ which form the set of alternatives. Suppose there exists three criterion $\mathrm{e}_{1}$ (greenery), $\mathrm{e}_{2}$ (cheap), $\mathrm{e}_{3}($ beautiful $)$ and $\mathrm{e}_{4}$ (good surroundings) that are taken into account in this problem. Now decision maker want to choose a apartments depending upon the criteria $\mathrm{e}_{1}, \mathrm{e}_{2}, \mathrm{e}_{3}$ or $\mathrm{e}_{4}$. Let the observations made are expressed as follows:

Step 1: Construct a IFSS (F,E) over U, which can be prepared with the help of apartment association.

\begin{tabular}{|c|c|c|c|c|}
\hline$(\mathrm{F}, \mathrm{E})$ & $\mathrm{e}_{1}$ & $\mathrm{e}_{2}$ & $\mathrm{e}_{3}$ & $\mathrm{e}_{4}$ \\
\hline $\mathrm{a}_{1}$ & $(0.3,0.6)$ & $(0.7,0.2)$ & $(0.6,0.3)$ & $(0.5,0.3)$ \\
\hline $\mathrm{a}_{2}$ & $(0.6,0.1)$ & $(0.6,0.2)$ & $(0.7,0.0)$ & $(0.1,0.4)$ \\
\hline
\end{tabular}

Step 2: Construct a IFSS (G,E) First apartments under consideration.

\begin{tabular}{|c|c|c|c|c|}
\hline$(\mathrm{G}, \mathrm{E})$ & $\mathrm{e}_{1}$ & $\mathrm{e}_{2}$ & $\mathrm{e}_{3}$ & $\mathrm{e}_{4}$ \\
\hline $\mathrm{a}_{1}$ & $(0.2,0.1)$ & $(0.2,0.1)$ & $(0.3,0.1)$ & $(0.2,0.1)$ \\
\hline $\mathrm{a}_{2}$ & $(0.2,0.1)$ & $(0.3,0.1)$ & $(0.2,0.0)$ & $(0.3,0.3)$ \\
\hline
\end{tabular}




\section{International Journal of Science and Research (IJSR) \\ ISSN (Online): 2319-7064}

Index Copernicus Value (2013): 6.14 | Impact Factor (2015): 6.391

Step: 3 Construct IFSS (H, E) over the universe $U$ for the second Apartments.

\begin{tabular}{|c|c|c|c|c|}
\hline$(\mathrm{H}, \mathrm{E})$ & $\mathrm{e}_{1}$ & $\mathrm{e}_{2}$ & $\mathrm{e}_{3}$ & $\mathrm{e}_{4}$ \\
\hline $\mathrm{a}_{1}$ & $(0.6,0.2)$ & $(0.4,0.2)$ & $(0.4,0.2)$ & $(0.4,0.4)$ \\
\hline $\mathrm{a}_{2}$ & $(0.4,0.2)$ & $(0.5,0.4)$ & $(0.6,0.1)$ & $(0.7,0.1)$ \\
\hline
\end{tabular}

Step: 4 Calculate similarity measure between $(\mathrm{F}, \mathrm{E})$ and $(\mathrm{G}, \mathrm{E}) ;(\mathrm{F}, \mathrm{E})$ and $(\mathrm{H}, \mathrm{E})$

$$
\begin{aligned}
&\left.\mathrm{M}_{1}((\mathrm{~F}, \mathrm{E}),(\mathrm{G}, \mathrm{E}))=0.25 ; \mathrm{M}_{1}(\mathrm{~F}, \mathrm{E}),(\mathrm{H}, \mathrm{E})\right)=0.56 \\
& \mathrm{M}_{2}((\mathrm{~F}, \mathrm{E}),(\mathrm{G}, \mathrm{E}))=0.33 ; \mathrm{M}_{2}((\mathrm{~F}, \mathrm{E}),(\mathrm{H}, \mathrm{E}))=0.34 \\
& \mathrm{M}_{3}((\mathrm{~F}, \mathrm{E}),(\mathrm{G}, \mathrm{E}))=0.40 ; \mathrm{M}_{3}((\mathrm{~F}, \mathrm{E}),(\mathrm{H}, \mathrm{E}))=0.70 \\
&\left.\mathrm{M}_{4}((\mathrm{~F}, \mathrm{E}),(\mathrm{G}, \mathrm{E}))=0.20 ; \mathrm{M}_{4}(\mathrm{~F}, \mathrm{E}),(\mathrm{H}, \mathrm{E})\right)=0.35 \\
& \mathrm{M}(\mathrm{F}, \mathrm{G})=\operatorname{Max}\left\{\mathrm{M}_{1}, \mathrm{M}_{2,} \mathrm{M}_{3}, \mathrm{M}_{4}\right\} \\
&= \operatorname{Max}\{0.25,0.33,0.4,0.2\}=0.4<1 / 2 \\
& \mathrm{M}(\mathrm{F}, \mathrm{H})=\operatorname{Max}\left\{\mathrm{M}_{1}, \mathrm{M}_{2,} \mathrm{M}_{3}, \mathrm{M}_{4}\right\} \\
&=\operatorname{Max}\{0.56,0.34,0.7,0.35\}=0.7>1 / 2
\end{aligned}
$$

$\mathrm{M}(\mathrm{F}, \mathrm{G})=0.40<1 / 2 ; \mathrm{M}(\mathrm{F}, \mathrm{H})=0.70>1 / 2$

Therefore we conclude that the second apartment is the best apartment in the town.

\section{Conclusion}

In this paper we studied the algebraic properties of intuitionistic fuzzy soft sets .We have made an investigation on existing basic notions and results on intuitionistic fuzzy soft sets. Next we define similarity measure between two IFSS based on set theoretical approach then we construct a Similarity measure between two IFSS based on set theoretical approach. Finally we give an example to show the possibilities of applications of similarity measure between two IFS sets in a decision making problem. Thus we can use the method to solve the problems that contain uncertainty such as problem in social, economic system, pattern recognition, medical diagnosis, and so on.

\section{References}

[1] B.Ahmad and AtharKharal, "On Fuzzy Soft Sets", Advances in Fuzzy Systems, 2009.

[2] K. Atanassov, "Intuitionistic fuzzy sets", Fuzzy Sets and Systems 20 ( 1986 ), 87-96.

[3] K.T., Atanassov, Intuitionistic Fuzzy Sets- Theory and Applications, Physica-Verlag, A Springer-Verlag Company, New York (1999).

[4] N.,Cagman and S., Karatas, Intuitionistic Fuzzy Soft set theory and its decision making, Journal of Intelligent and fuzzy Systems, 24(2013) 829-836.

[5] N.,Cagman and S., Enginoglu , Fuzzy Soft Sets theory and its Applications, Iranian Journal of Fuzzy Systems, 8(3)(2011) 137-147.

[6] B. Chetia, P. K. Das, "Some Results of Intuitionistic Fuzzy Soft Sets and its Application in Decision Making", Applied Mathematical Sciences, Vol. 7, 2013, no. 95,4693 - 4712

[7] P.K.Maji, R.Biswas and A.R.Roy, "Fuzzy Soft Sets", Journal of Fuzzy Mathematics,9(3), 2001, 589-602.

[8] P.K.Maji, R.Biswas, A.R.Roy, "Intuitionistic fuzzy soft sets", The journal of fuzzy mathematics 9(3)( 2001 ), 677-692.
[9] P.K., Maji., R., Biswas, and A.R., Roy, On Intuitionistic Fuzzy Soft Sets., The Journal of Fuzzy Mathematics, 12(3) (2004) 669-683.

[10] Manoj Bora, Tridiv Jyoti Neog, Dusmanta Kumar Sut," Some Results on Intuitionistic Fuzzy Soft Sets", International Journal of Mathematics Trends and Technology - Volume3 Issue2- 2012, ISSN: 2231-5373

[11] Manoj Bora, Tridiv Jyoti Neog, Dusmanta Kumar Sut, "Some New Operations of Intuitionistic Fuzzy Soft Sets", International Journal of Soft Computing and Engineering (IJSCE) ISSN: 2231-2307, Volume-2, Issue-4, September 2012

[12]D. Molodtsov, "Soft Set Theory - First Results", Comput. Math. Appl. No.37, PP 1931, 1999.

[13]L.A.,Zadeh, Fuzzy sets,Inform. Control 8 (1965) 338353.

Volume 5 Issue 6, June 2016 www.ijsr.net

Licensed Under Creative Commons Attribution CC BY 